\title{
BMJ Open The Malaysian Breast Cancer Survivorship Cohort (MyBCC): a study protocol
}

Tania Islam, ${ }^{1,2}$ Nirmala Bhoo-Pathy, ${ }^{3}$ Tin Tin Su, ${ }^{1}$ Hazreen Abdul Majid, ${ }^{1}$ Azmi Mohd Nahar, ${ }^{4}$ Chong Guan Ng, ${ }^{5}$ Maznah Dahlui, ${ }^{1}$ Samsinah Hussain, ${ }^{6}$ Marie Cantwell, ${ }^{7}$ Liam Murray, ${ }^{7}$ Nur Aishah Taib, ${ }^{2}$ MyBCC study group

To cite: Islam T, BhooPathy N, Su TT, et al. The Malaysian Breast Cancer Survivorship Cohort (MyBCC): a study protocol. BMJ Open 2015;5:e008643. doi:10.1136/bmjopen-2015008643

- Prepublication history for this paper is available online. To view these files please visit the journal online (http://dx.doi.org/10.1136/ bmjopen-2015-008643)

Received 30 April 2015 Revised 16 August 2015 Accepted 25 August 2015

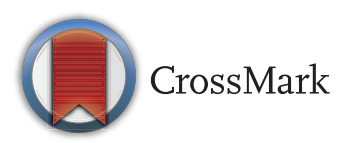

For numbered affiliations see end of article.

Correspondence to Professor Nur Aishah Taib; naisha@um.edu.my

\section{ABSTRACT}

Introduction: Over recent decades, the burden of breast cancer has been increasing at an alarming rate in Asia. Prognostic research findings from Western countries may not readily be adapted to Asia, as the outcome of breast cancer depends on a multitude of factors ranging from genetic, clinical and histological predictors, to lifestyle and social predictors. The primary aim of this study is to determine the impact of lifestyle (eg, nutrition, physical activity), mental and sociocultural condition, on the overall survival and quality of life (QoL) among multiethnic Malaysian women following diagnosis of breast cancer. This study aims to advance the evidence on prognostic factors of breast cancer within the Asian setting. The findings may guide management of patients with breast cancer not only during active treatment but also during the survivorship period.

Methods: This hospital-based prospective cohort study will comprise patients with breast cancer (18 years and above), managed in the University Malaya Medical Centre (UMMC). We aim to recruit 1000 cancer survivors over a 6 -year period. Data collection will occur at baseline (within 3 months of diagnosis), 6 months, and 1, 3 and 5 years following diagnosis. The primary outcomes are disease-free survival and overall survival, and secondary outcome is QoL. Factors measured are demographic and socioeconomic factors, lifestyle factors (eg, dietary intake, physical activity), anthropometry measurements (eg, height, weight, waist, hip circumference, body fat analysis), psychosocial aspects, and complementary and alternative medicine (CAM) usage.

Ethics and dissemination: This protocol was approved by the UMMC Ethical Committee in January 2012. All participants are required to provide written informed consent. The findings from our cohort study will be disseminated via scientific publication as well as presentation to stakeholders including the patients, clinicians, the public and policymakers, via appropriate avenues.

\section{BACKGROUND}

The past two decades observed a steep increase in breast cancer incidence in most Asian countries. ${ }^{1-4}$ However, this finding is in contrast to Western countries, where breast cancer incidence has stabilised or even decreased. ${ }^{5-7}$ While early detection and new treatment regimes have both contributed to significant improvement in breast cancer survival rates in developed countries, ${ }^{8}$ mortality rates due to breast cancer have been escalating in most parts of Asia. ${ }^{9}{ }^{10}$ Amidst economic improvement and westernisation of Asian countries, distinct changes have been noticed in the lifestyle of women, in their reproductive factors and in the amount of exposure to environmental toxins. ${ }^{11-13}$ Although it has been established that risk factors for breast cancer in Asian populations are similar to those in Western populations, the prognostic factors may be different from those in Western countries; these include ethnicity, physical activity, obesity, as well as nutritional factors/dietary intake. ${ }^{1} 11{ }^{14}$ In addition, the diverse Asian culture, different religions, varied ethnicity and lifestyles may also influence patients with breast cancer and affect their survivorship and quality of life (QoL) ${ }^{15}{ }^{16}$ Body fatness has been frequently associated with increased risk of breast cancer and lower subsequent survival in the West, ${ }^{17}$ but little is known on the possible impact of obesity on survival following breast cancer within Asian settings. Breast cancer survivors also tend to be younger in Asia than survivors in Western countries. ${ }^{18}$ Thus the factors associated with breast cancer and survival in Asian countries may differ from those in Western countries. It is therefore intriguing to understand the impact of lifestyle, health behaviour, genetics, tumour biology and psychosocial factors, on QoL and overall survival of women from different ethnic groups, diagnosed with breast cancer in Asian settings.

Breast cancer is the most common cancer among Malaysian women. ${ }^{19}$ Malaysia is a multiethnic country comprising of three 
major ethnic groups. The largest proportion of the population is represented by Malays, subsequently followed by Chinese and Indians. ${ }^{20}{ }^{21}$ Age-standardised incidence rate of breast cancer is highest among the Chinese, followed by Indians and lastly by Malays. However, there is evidence that the Malay ethnic group had lowest 5-year overall survival compared to the Indian and Chinese groups. In multiethnic South East Asian settings, survival of women with breast cancer seems to be associated with ethnicity, independent of stage at diagnosis, tumour pathology and treatment. ${ }^{20-22}$ Since ethnicity and culture interact with lifestyle choices in Malaysia, breast cancer survivors can be a good source to identify associations among these factors, in order to study the impact of these factors on survival and other outcomes. Likewise, this study will also establish probable associations between lifestyle and psychosocial condition on disease-free survival, overall survival and QoL after diagnosis of breast cancer. As such, the Malaysian Breast Cancer Survivorship Cohort (MyBCC) study is expected to provide evidence to improve the understanding of factors influencing breast cancer survivorship in multiethnic settings, and hence guide the management of breast cancer survivors.

\section{OBJECTIVES}

The primary objective of our study is to determine the association between demographic, socioeconomic status, lifestyle factors (dietary intake, physical activity), body composition, psychosocial factors, return to work (RTW), as well as complementary and alternative medicine (CAM) use, and overall survival as well as quality of life (QoL) among multiethnic breast cancer survivors.

\section{Specific objectives}

1. To identify the ethnic differences in survival and QoL among Malaysian women after a diagnosis of breast cancer.

2. To assess the impact of socioeconomic inequality on survival and QoL among Malaysian breast cancer survivors.

3. To determine if (change of) body composition impacts survival and QoL of Malaysian women with breast cancer.

4. To study nutritional status and dietary intake among breast cancer survivors and their impact on survival and QoL.

5. To determine the level of physical activity and its effect on survival and QoL among breast cancer survivors.

6. To determine the prevalence and determinants of CAM use and its impact on survival and QoL based on breast cancer survivors.

7. To determine the level of distress, anxiety and depression, and its effect on survival and QoL among breast cancer survivors.
8. To assess financial difficulty and RTW rates and their effect on QoL among breast cancer survivors.

We hypothesise that demography, socioeconomic status, lifestyle (ie, nutrition, physical activity), body composition, work-related and psychosocial factors, and CAM use of the different ethnic groups, will impact the overall survival and QoL of Malaysian patients with breast cancer.

\section{METHODS}

\section{Study design}

This hospital-based prospective cohort study will include those newly diagnosed patients with breast cancer (aged 18 years and above) managed in University Malaya Medical Centre (UMMC), Malaysia, who provide written-informed consent to participate. The UMMC is a tertiary academic hospital, situated in a relatively affluent part of Kuala Lumpur, which caters to a predominantly middle-class urban population. In 1993, this hospital established a prospective breast cancer registry. Based on a report of the National Cancer Registry in 2006, UMMC managed approximately $10 \%$ of newly diagnosed patients with breast cancer in Malaysia. For the current study, data on demography, clinical profile, pathological tumour characteristics and treatment will be obtained from the breast cancer registry. A baseline anthropometric measurement and body fat analysis (BFA) will be conducted by trained research assistants within 3 months of diagnosis. Figure 1 demonstrates the procedure of study recruitment and the overall flow of study. A survey in the form of a questionnaire will be conducted by trained interviewers at around the time of diagnosis (baseline) and at half year, first year, third year and fifth year follow-up, in order to collect data on QoL, socioeconomic status, physical activity, psychosocial support, RTW duration, and CAM usage among breast cancer survivors. A baseline assessment of dietary intake among survivors will be made using a 3-day food diary approximately 1 year after diagnosis. The rationale being to observe survivors' habitual dietary intake after they are back to their normal life after completion of all conventional treatment. The survivors' dietary intake assessment will be subsequently repeated at the third and fifth years after diagnosis (figure 1).

\section{Study participants}

This study will recruit patients with breast cancer diagnosed and managed in the UMMC since February 2012 and will continue recruiting until February 2017. The participants will be followed up at 6 months, and 1,3 and 5 years after the date of recruitment, and this will continue until 2022. All newly diagnosed patients with breast cancer in the UMMC breast clinic will be screened for eligibility. Only eligible patients who give consent will be recruited. Figure 1 shows detailed information on our recruitment strategy. 
Figure 1 Flow chart of study recruitment process and overall study design (BFA, body fat analysis; BMI, body mass index; UMMC, University Malaya Medical Centre; WHR, waist-to-hip ratio).
Newly diagnosed breast cancer patients at UMMC breast clinic

Screening

Eligible patients in Surgery and oncology clinic

Consent given

Administration of baseline questionnaire, and collection of socio-demographic and clinical data Physical measurement (BMI, WHR and BFA)

Collection of biospecimen (blood, urine, and tissues)

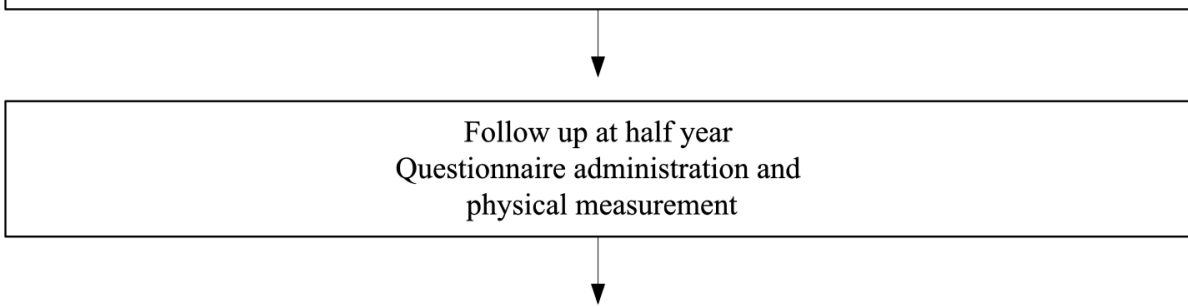

Follow up at 1 st year

Questionnaire and food diary administrations,

Physical measurement, and collection of blood

Follow-up at 5 th year

Questionnaire, and food diary administration,

Physical measurement
Eligibility criteria

Inclusion criteria

All women who are newly diagnosed with primary breast cancer in UMMC:

- Aged 18 years and above

- Diagnosed between February 2012 and December 2017

- Within 3 months of diagnosis

- TNM stage I-IV breast cancer

- Malaysian

- Able to converse either in Malay, English, Mandarin or Tamil

\section{Exclusion criteria}

- Patients with prior history of any other cancer

- Patients whose attending physician certifies them as unfit due to other prevailing medical condition

- Bedridden at time of recruitment

\section{Ethics and dissemination}

The study protocol and procedures were approved by the UMMC Ethical Committee (MEC number 896.150).
We obtained full permission from the European Organization for Research and Treatment of Cancer (EORTC) to use the QoL questionnaires, EORTC QLQ-C30 and QLQ-BR23. Ethical approval was obtained from University Malaya Cancer Research Institute (MEC number 775.9) for collection of biospecimens. Eligible patients will be verbally informed, by trained research personnel, regarding the nature and purpose of the study, and given time to decide whether or not to participate. Written informed consent will be obtained from study participants prior to their enrolment and all collected information will be treated as confidential. We intend to present the results of our cohort study via scientific publication (peer-reviewed) as well as through presentation to stakeholders including the public, patients, clinicians and policymakers, via appropriate avenues.

\section{Measures}

\section{Exposure measures}

The exposures of interest are demographic variables (eg, age at diagnosis, ethnicity, age at marriage, age at 
first child birth, age at last child birth), socioeconomic variables (income, highest attained-education and occupation), anthropometric measurements (eg, height, weight, waist hip circumference, percentage of body fat), diet (habitual food intake), use of CAM (eg, used types of treatment other than hospital treatment), physical activity, work-related variables (type of work, working hours), health-related variables (eg, physical condition, appetite), psychosocial variables (eg, anxiety, depression) and biomarkers (eg, blood, tumour tissues).

Data on the exposure of interest will be obtained through personal interview (face-to-face), food diaries, and anthropometric and biological measurement, details of which are given in table 1 .

\section{Outcome measures}

Patients will be monitored through follow-up in the breast cancer outpatient clinics in UMMC. Patients who are unable to attend clinics and those who are not scheduled for regular follow-up in UMMC will be contacted by phone.

The primary outcome is overall survival. Data on mortality will be obtained from the hospital's medical records, as well as active follow-up through the next-of-kin of patients. In addition, we will regularly update the vital status of patients through direct linkage with the National Registration Department (NRD), Malaysia, which holds the death records in Malaysia.

The cohort will be followed up with scheduled phone calls and clinic visits in a systematic way to update patient's health status. Another outcome is QoL. QoL will be measured based on response to a QoL questionnaire obtained from the EORTC. The EORTC QLQ-C30 is comprised of 30 items including five functional scales (physical, role, emotional, cognitive and social) and 9 symptom scales (fatigue, nausea and vomiting, pain, dyspnoea, insomnia, appetite loss, constipation, diarrhoea, and financial difficulties) and Global health status scale. On the contrary, the QLQ-BR23 is a more detailed questionnaire, containing 23 questions designed to quantify the QoL of patients with breast cancer. It includes four functional scales (body image, sexual functioning, sexual enjoyment, future perspective) and four symptom scales (systemic therapy side effect, breast symptom, arm symptom, upset by hair loss). Information from the questionnaires will be scored accordingly. The raw score for each subscale will be calculated and subsequently linearly transformed to a level between 0 and 100 (standardised raw score) according to the guidelines of the EORTC scoring manual. A higher score for functional scale represents a high/healthy level of functioning. A high score for the global health status/ QoL represents high QoL. However, a high score for a symptom scale represents a high level of symptom, indicating a worse QoL.

\section{Tools used for measures}

1. Anthropometry: Body mass index (BMI), body fat percentage and waist-to-hip ratio (WHR) will be used as indicators of body fatness. WHO definitions and classifications will be used. Patients' nutritional status will be measured from BMI, BFA, WHR and food diaries. BMI and BFA will be measured at baseline, and during subsequent follow-up at 6 months, and 1, 3

Table 1 Measurement domains and data collection time points

\begin{tabular}{|c|c|c|c|c|c|c|}
\hline Theme & Instruments & Baseline & 6 Months & 1 Year & 3 Years & 5 Years \\
\hline \multirow{2}{*}{$\begin{array}{l}\text { Demographic and } \\
\text { socioeconomic } \\
\text { characteristics }\end{array}$} & Sociodemographic & $x$ & & & & \\
\hline & Socioeconomy background (questionnaire) & $x$ & $x$ & & & \\
\hline \multirow[t]{2}{*}{ Quality of life } & $\begin{array}{l}\text { European Organization for Research and } \\
\text { Treatment of Cancer Core Quality of Life } \\
\text { Questionnaire (EORTC QLQ-C30) }\end{array}$ & $x$ & $x$ & $x$ & $x$ & $\times$ \\
\hline & $\begin{array}{l}\text { EORTC of Cancer Breast Cancer Quality of } \\
\text { Life Questionnaire (QLQ-BR23) }\end{array}$ & $x$ & $x$ & $x$ & $\times$ & $x$ \\
\hline \multirow[t]{2}{*}{$\begin{array}{l}\text { Body weight and } \\
\text { nutrition }\end{array}$} & $\begin{array}{l}\text { Anthropometric measurement (body mass } \\
\text { index, waist-to-hip ratio) }\end{array}$ & $x$ & $x$ & $x$ & $x$ & $x$ \\
\hline & Food diary and mini-food album & & & $x$ & $x$ & $\times$ \\
\hline Physical activity & Global Physical Activity Questionnaire (GPAQ) & $\times$ & $x$ & $x$ & $x$ & $\times$ \\
\hline $\begin{array}{l}\text { Complementary and } \\
\text { alternative medicine } \\
\text { (CAM) }\end{array}$ & CAM questionnaire & $x$ & $\times$ & $x$ & $x$ & $\times$ \\
\hline \multirow[t]{2}{*}{ Psychosocial factors } & $\begin{array}{l}\text { Hospital Anxiety and Depression Score } \\
\text { (HADS) and }\end{array}$ & $x$ & $x$ & $x$ & $x$ & $\times$ \\
\hline & Distress Thermometer (questionnaire) & $\times$ & $x$ & $x$ & $x$ & $\times$ \\
\hline \multirow[t]{2}{*}{ Work-related factors } & Return to work self-efficacy scale (RTW-SE) & $\times$ & $x$ & $\times$ & $\times$ & $\times$ \\
\hline & Readiness for RTW scale & $x$ & $x$ & $x$ & $x$ & $\times$ \\
\hline \multirow{2}{*}{$\begin{array}{l}\text { Clinical, histological } \\
\text { and biological factors }\end{array}$} & Clinical registry & $x$ & $x$ & $x$ & $x$ & $\times$ \\
\hline & Biobank (blood, urine and tissues) & $x$ & & $\begin{array}{l}\times \text { (only } \\
\text { blood) }\end{array}$ & & \\
\hline
\end{tabular}


and 5 years. WHR will be measured only at baseline. BMI and BFA, body fat percentages, will be measured using a portable body composition analyser (TANITA BC-418 Body Composition Analyser, Tokyo, Japan). This equipment will calculate body fat ratio, body fat mass, fat-free mass, estimated muscle mass and Basal Metabolic Rate, using data derived using Bioelectric impedance analysis (BIA). Hydration status, recent physical activity, consumption of food or beverages, temperature, menstrual status and body position are among the factors that can affect the validity and precision of the measurements. For example, participants should avoid alcohol and vigorous exercise for $12 \mathrm{~h}$ before testing, so that body fluids are not affected prior to the measurements. The measurements should be taken on participants approximately $3 \mathrm{~h}$ after eating, and within $30 \mathrm{~min}$ of voiding.

2. Food diary: Habitual food intake will be assessed using a self-administered 3-day food diary. A qualified trained dietician will provide information and give instructions on how to complete the 3-day dietary records. The food diary will be generalised to all ethnic groups (Malays, Chinese and Indians). A minifood album detailing (multiethnic) Malaysian food items will be provided for guidance. Participants will be reassessed at 2 and 4 years after initial dietary assessment.

3. Questionnaire: Questionnaires will be used to obtain detailed information on concurrent illness, workrelated factors, CAM use, physical activity and QoL $^{23-25}$ (described in detail in table 1). Questionnaires for RTW self-efficacy scale (RTW-SE), readiness for RTW scale and CAM use have undergone linguistic, content and face validation. Physical activity will be measured using the Global Physical Activity Questionnaire (GPAQ). Presence of anxiety and depression will be assessed using the Hospital Anxiety and Depression Score (HADS). To measure QoL, two modules will be used: EORTC QLQ-C30 and EORTC QLQ-BR23, which have been translated to the local languages and validated. The validity and reproducibility of EORTC QLQ-C30, GPAQ and HADS have been proven satisfactory. ${ }^{26-28}$

4. Clinical registry: There is already an established prospective breast cancer registry in UMMC, ${ }^{11}$ which includes: data on reproductive factors and family history; tumour characteristics, namely, size, grade, TNM stage, receptor status (oestrogen receptor, progesterone receptor, human epidermal growth factor receptor 2); and treatment details, those being treatment modalities (surgery, radiotherapy, chemotherapy and hormonal therapy). We will regularly link the current study variables to the registry.

\section{Data collection point}

Baseline

At the time of recruitment to the study, the following information will be recorded:
- Demographic factors and clinical data

- Information on other illnesses/comorbidities

- Details on

- QoL

- Psychosocial factors

- Work-related factors

- Physical activity

- CAM use

- Biospecimens collected (blood, urine and tissues) will be preserved

BMI, WHR and BFA measurement

\section{Follow-up}

The following information will be collected at 6 months, and 1, 3 and 5 years after diagnosis:

- Clinical data and particulars on concurrent illness

- Details on

- QoL

- Psychosocial factors

- Physical activity

- CAM use

- Work-related factors

- Food diaries (during 1st, 3rd and 5th year follow-up period)

- BMI and BFA measurement

Figure 1 demonstrates the overall flow of the study.

\section{DATA ANALYSIS}

\section{Statistical analysis}

The results of survivors' demographics and baseline outcome variables will be summarised using descriptive summary measures, expressed as mean or median for continuous variables and proportions for categorical variables. We will report the model coefficient for continuous outcomes, OR for binary outcomes, or HR for time to event outcomes, with the corresponding 95\% CIs, and associated $\mathrm{p}$ values. To determine the QoL among patients with breast cancer at different stages of the illness, we will use scores obtained from the EORTC QLQ-C30 and EORTC QLQ-BR23 instruments. A higher score represents a 'better' level of functioning and global health status, or a 'worse' level of symptoms. Median and other quartiles with $95 \%$ CI will be obtained for all the domains of QoL index according to the stage of breast cancer. The analysis of variance test will be conducted to determine the QoL score for each domain, according to respective cancer stages and ethnicity, $\mathrm{p}$ value $<0.05$ will be considered to be statistically significant.

Important prognostic factors will be identified by fitting the Cox's proportional hazard model. Overall survival will be estimated using the Kaplan-Meier method and compared using the log-rank test. Multivariable Cox regression analysis will be used to estimate the relative risk of all-cause mortality (HR) associated with the various prognostic factors that we are studying. We will also assess whether ethnicity modifies the association 
between various prognostic factors and overall survival, as well as QoL.

\section{Statistical power}

We estimate that we will be able to recruit about 1000 women during the 6 years of enrolment, that is, $150-200$ patients per year in UMMC. With reports showing Malaysia to have the highest prevalence of obesity in Asia, ${ }^{29}$ we hypothesise that change in body weight may explain most of the survival disparities associated with lifestyle. Previous studies in UMMC recorded a mean BMI of patients with breast cancer at diagnosis as $23.8 \mathrm{~kg} / \mathrm{m}^{2} \quad(\mathrm{SD}=4.12)$ and overall 5-year survival at $75 \% .{ }^{20}{ }^{21}$ A Cox regression of the $\log$ HR on BMI of these women with an SD of $4 \mathrm{~kg} / \mathrm{m}^{2}$ based on a sample of 1000 participating women achieves an output of $90 \%$ statistical power at a 5\% significance level to detect a regression coefficient of BMI $\left(\mathrm{kg} / \mathrm{m}^{2}\right)$ equivalent to 0.0468. Recruitment of even 750 patients will be able to achieve $80 \%$ statistical power at $5 \%$ significance to detect a significant difference.

\section{DISCUSSION}

Prognoses of breast cancer among Asian women vary considerably from those of Western women and may be attributable to difference in lifestyle, socioeconomic profile, health beliefs, culture and genetic backgrounds. ${ }^{10}$ Previous studies in selected populations (Malaysian) have suggested that ethnicity is an independent prognostic factor of breast cancer survival. ${ }^{21}$ Development of biomarkers for prognosis, an expanding biobank and, furthermore, outcome measures and QoL measures among cancer survivors, are relatively scarce in Asia.

To the best of our knowledge, this study is the first, in a multiethnic Asian setting, to determine the association between sociodemographic and lifestyle factors on overall survival and QoL in breast cancer survivors. Notably, previous cohort studies ${ }^{11}{ }^{30}$ only conducted survival analysis utilising clinical data. Results from our study can provide insight into association between patients' nutrition, BMI, body composition, physical, mental and socioeconomic status, QoL and overall survival. We will also be able to assess the influence of ethnicity on these associations. A major strength of this study is that we are using well-validated and reliable tools (ie, GPAQ and portable TANITA) ${ }^{31-33}$ to measure modifiable risk factors (ie, physical activity, body composition). This study will add evidence on the impact of body fatness and its changes on overall survival and QoL of Asian women following the diagnosis of breast cancer. In addition, there will be longterm storage of patients' blood, serum, plasma, urine and tissues in the UMMC Biobank, for future research.

There are some limitations to the current study. It is expected that there will be some ambiguity in assessing the overall improvement or decrement in QoL of the survivors, as perception varies from one individual to another. Nevertheless, the validity and reproducibility of QoL has been satisfactory ${ }^{27}$ and was adopted from the EORTC. Given that we have chosen to address a cohort comprising mostly of urban residents attending a tertiary hospital, our findings may not necessarily reflect the overall situation of breast cancer survivors in Malaysia. Another limitation is that we will be using a 3-day food diary that depends on the respondent's full co-operation. However, diet history will be taken from 2 weekdays and 1 weekend day and, as such, should reflect more valid estimates of patients' habitual dietary intakes. ${ }^{34}$

On the basis of the cohort study proposed here, this knowledge will be important for developing an effective strategy for the improvement of overall survival and QoL of patients with breast cancer in middle-income countries such as Malaysia, where resources for healthcare are limited. This study will enable relevant parties to address prognostic factors that affect survival and enable a thorough focus to be placed on differences among ethnicity. Hence, we hope results from our study will contribute to the advancement in evaluation and appraisal methods for assessment of prognostic factors in breast cancer. In the long run, we aim to improve the survival and QoL following the diagnosis of breast cancer in Asian women.

\section{Author affiliations}

${ }^{1}$ Faculty of Medicine, Centre for Population Health (CePH) and Department of Social and Preventive Medicine, University of Malaya, Kuala Lumpur, Malaysia ${ }^{2}$ Faculty of Medicine, Department of Surgery, University of Malaya, Kuala Lumpur, Malaysia

${ }^{3}$ Faculty of Medicine, Julius Centre University of Malaya, University of Malaya, Kuala Lumpur, Malaysia

${ }^{4}$ Faculty of Medicine, Department of Sports Medicine, University of Malaya, Kuala Lumpur, Malaysia

${ }^{5}$ Department of Psychological Medicine, University Malaya Medical Centre, Kuala Lumpur, Malaysia

${ }^{6}$ Department of Pharmacy, Faculty of Medicine, University of Malaya, Kuala Lumpur, Malaysia

${ }^{7}$ Centre for Public Health, Queen's University of Belfast, Belfast, Ireland

Acknowledgements The authors thank the doctors, nurses, technical staff and hospital administration staff at UMMC for the daily administration of the Malaysian Breast Cancer Survivorship Cohort Study.

Collaborators Members of MyBCC working group: Taib NA, Bhoo-Pathy N, Su TT, Majid HA, Nahar AM, Ng CG, Dahlui M, and Hussain S, from University of Malaya, and Cantwell M, and Murray L from Queen's University Belfast.

Contributors NAT, TI, NB-P and TTS designed the study and drafted the manuscript. MD, LM, HAM and AMN made substantial contributions to the conception and design of the project. CGN, MC and SH were involved in drafting the manuscript. TI and NB-P were in charge of the statistical analysis. All the authors critically revised the manuscript for important intellectual content and provided approval of the final version to be published.

Funding The post-doctoral research fellow position for this project (UMQUB 3C-2011) was jointly funded by University of Malaya and Queen's University of Belfast. The study was supported by a High Impact Research Grant (UM.C/ HIR/MOHE/06) from the Ministry of Higher Education, Malaysia. 
Competing interests None declared.

Ethics approval The protocol was approved by the local ethics committee of UMMC, in January 2012.

Provenance and peer review Not commissioned; externally peer reviewed.

Data sharing statement Further information such as statistical examination can be obtained from the first author.

Open Access This is an Open Access article distributed in accordance with the Creative Commons Attribution Non Commercial (CC BY-NC 4.0) license, which permits others to distribute, remix, adapt, build upon this work noncommercially, and license their derivative works on different terms, provided the original work is properly cited and the use is non-commercial. See: http:// creativecommons.org/licenses/by-nc/4.0/

\section{REFERENCES}

1. Hirabayashi $\mathrm{Y}$, Zhang $\mathrm{M}$. Comparison of time trends in breast cancer incidence (1973-2002) in Asia, from cancer incidence in five continents, vols IV-IX. Jpn J Clin Oncol 2009;39:411-12.

2. Medina VM, Laudico A, Mirasol-Lumague MR, et al. Cumulative incidence trends of selected cancer sites in a Philippine population from 1983 to 2002: a joinpoint analysis. Br J Cancer 2010;102:1411-14.

3. Sim X, Ali RA, Wedren S, et al. Ethnic differences in the time trend of female breast cancer incidence: Singapore, 1968-2002. BMC Cancer 2006;6:261.

4. Takiar R, Srivastav A. Time trend in breast and cervix cancer of women in India-(1990-2003). Asian Pac J Cancer Prev 2008;9:777-80.

5. Fontenoy AM, Leux C, Delacour-Billon S, et al. Recent trends in breast cancer incidence rates in the Loire-Atlantique, France: a decline since 2003. Cancer Epidemiol 2010;34:238-43.

6. Ravdin PM, Cronin KA, Howlader N, et al. The decrease in breastcancer incidence in 2003 in the United States. N Engl J Med 2007;356:1670-4.

7. Glass AG, Lacey JV Jr, Carreon JD, et al. Breast cancer incidence, 1980-2006: combined roles of menopausal hormone therapy, screening mammography, and estrogen receptor status. J Natl Cancer Inst 2007;99:1152-61.

8. Coleman MP, Quaresma M, Berrino F, et al., CONCORD Working Group. Cancer survival in five continents: a worldwide population-based study (CONCORD). Lancet Oncol 2008;9:730-56.

9. Leong SP, Shen ZZ, Liu TJ, et al. Is breast cancer the same disease in Asian and Western countries? World J Surg 2010;34:2308-24.

10. Bhoo-Pathy N, Yip CH, Hartman M, et al. Breast cancer research in Asia: adopt or adapt Western knowledge? Eur J Cancer 2013;49:703-9.

11. Pathy NB, Yip CH, Taib NA, et al. Breast cancer in a multi-ethnic Asian setting: results from the Singapore-Malaysia hospital-based breast cancer registry. Breast 2011;20(Suppl 2):S75-80.

12. Coughlin SS, Ekwueme DU. Breast cancer as a global health concern. Cancer Epidemiol 2009;33:315-18.

13. Saxena N, Hartman M, Bhoo-Pathy N, et al. Breast cancer in South East Asia: comparison of presentation and outcome between a middle income and a high income country. World J Surg 2012;36:2838-46.

14. Islam $T$, Ito $H$, Sueta $A$, et al. Alcohol and dietary folate intake and the risk of breast cancer: a case-control study in Japan. Eur J Cancer Prev 2013;22:358-66.

15. Lin YH, Chiu JH. Use of Chinese medicine by women with breast cancer: a nationwide cross-sectional study in Taiwan. Complement Ther Med 2011;19:137-43.
16. Taib NA, Yip CH, Low WY. A grounded explanation of why women present with advanced breast cancer. World J Surg 2014;38:1676-84.

17. Kwan ML, John EM, Caan BJ, et al. Obesity and mortality after breast cancer by race/ethnicity: the California Breast Cancer Survivorship Consortium. Am J Epidemiol 2013;179:95-111.

18. Ahn E, Cho J, Shin DW, et al. Impact of breast cancer diagnosis and treatment on work-related life and factors affecting them. Breast Cancer Res Treat 2009;116:609-16.

19. Lim G, Rampal S, Halimah Y, In cancer incidence in Penisular Malaysia, 2003-2005 National Cancer Registry. Kuala Lumpur: National Cancer Registry, 2008.

20. Taib NA, Akmal M, Mohamed I, et al. Improvement in survival of breast cancer patients-trends in survival over two time periods in a single institution in an Asia Pacific Country, Malaysia. Asian Pacific $J$ Cancer Prev 2011;12:345-9.

21. Bhoo-Pathy N, Hartman M, Yip CH, et al. Ethnic differences in survival after breast cancer in South East Asia. PLoS ONE 2012;7: e30995.

22. Mohd Taib NA, Yip $\mathrm{CH}$, Mohamed I. Survival analysis of Malaysian women with breast cancer: results from the University of Malaya Medical Centre. Asian Pac J Cancer Prev 2008;9: 197-202.

23. Chen Z, Gu K, Zheng Y, et al. The use of complementary and alternative medicine among Chinese women with breast cancer. J Altern Complement Med 2008;14:1049-55.

24. Zigmond AS, Snaith RP. The hospital anxiety and depression scale. Acta Psychiatr Scand 1983;67:361-70.

25. Lagerveld SE, Bültmann U, Franche RL, et al. Factors associated with work participation and work functioning in depressed workers: a systematic review. J Occup Rehabil 2010;20:275-92.

26. Soo KL, Wan Abdul Manan WM, Wan Suriati WN, et al. The Bahasa Melayu Version of the Global Physical Activity Questionnaire: Reliability and Validity Study in Malaysia. Asia Pac J Public Health 2015;27:NP184-93.

27. Yusoff N, Low WY, Yip CH. The Malay Version of the European Organization for Research and Treatment of Cancer Quality of Life Questionnaire (EORTC-QLQ C30): Reliability and Validity Study. Int Med J Malays 2010;9:45-50.

28. Yusoff N, Low WY, Yip CH. Psychometric properties of the Malay Version of the hospital anxiety and depression scale: a study of husbands of breast cancer patients in Kuala Lumpur, Malaysia. Asian Pac J Cancer Prev 2011;12:915-17.

29. $\mathrm{Ng} \mathrm{M}$, Fleming $\mathrm{T}$, Robinson $\mathrm{M}$, et al. Global, regional, and national prevalence of overweight and obesity in children and adults during 1980-2013: a systematic analysis for the Global Burden of Disease Study 2013. Lancet 2014;384:766-81.

30. Abdullah NA, Wan Mahiyuddin WR, Muhammad NA, et al. Survival rate of breast cancer patients in Malaysia: a population-based study. Asian Pac J Cancer Prev 2013;14:4591-4.

31. Bull FC, Maslin TS, Armstrong T. Global physical activity questionnaire (GPAQ): nine country reliability and validity study. J Phys Act Health 2009;6:790-804.

32. Barreira TV, Staiano AE, Katzmarzyk PT. Validity assessment of a portable bioimpedance scale to estimate body fat percentage in white and African-American children and adolescents. Pediatr Obes 2013;8:e29-32.

33. Sim PY, Su TT, Abd Majid H, et al. A comparison study of portable foot-to-foot bioelectrical impedance scale to measure body fat percentage in Asian adults and children. Biomed Res Int 2014;2014:475659.

34. Cuenca-Garcia M, Artero EG, Sui X, et al. Dietary indices, cardiovascular risk factors and mortality in middle-aged adults: findings from the Aerobics Center Longitudinal Study. Ann Epidemiol 2014;24:297-303.e2. 